\title{
An efficient and improved method for virus-induced gene silencing in sorghum
}

\author{
Dharmendra Kumar Singh ${ }^{1}$, Hee-Kyung Lee ${ }^{1}$, Ismail Dweikat ${ }^{2}$ and Kirankumar S. Mysore ${ }^{1 *}$ (D)
}

\begin{abstract}
Background: Although the draft genome of sorghum is available, the understanding of gene function is limited due to the lack of extensive mutant resources. Virus-induced gene silencing (VIGS) is an alternative to mutant resources to study gene function. This study reports an improved and efficient method for Brome mosaic virus (BMV)-based VIGS in sorghum.

Methods: Sorghum plants were rub-inoculated with sap prepared by grinding $2 \mathrm{~g}$ of infected Nicotiana benthamiana leaf in $1 \mathrm{ml} 10 \mathrm{mM}$ potassium phosphate buffer (pH 6.8) and $100 \mathrm{mg}$ of carborundum abrasive. The sap was rubbed on two to three top leaves of sorghum. Inoculated plants were covered with a dome to maintain high humidity and kept in the dark for two days at $18^{\circ} \mathrm{C}$. Inoculated plants were then transferred to $18^{\circ} \mathrm{C}$ growth chamber with $12 \mathrm{~h} / 12 \mathrm{~h}$ light/dark cycle.
\end{abstract}

Results: This study shows that BMV infection rate can be significantly increased in sorghum by incubating plants at $18{ }^{\circ} \mathrm{C}$. A substantial variation in BMV infection rate in sorghum genotypes/varieties was observed and BTx623 was the most susceptible. Ubiquitin (Ubiq) silencing is a better visual marker for VIGS in sorghum compared to other markers such as Magnesium Chelatase subunit $H$ (Ch/H) and Phytoene desaturase (PDS). The use of antisense strand of a gene in BMV was found to significantly increase the efficiency and extent of VIGS in sorghum. In situ hybridization experiments showed that the non-uniform silencing in sorghum is due to the uneven spread of the virus. This study further demonstrates that genes could also be silenced in the inflorescence of sorghum.

Conclusion: In general, sorghum plants are difficult to infect with BMV and therefore recalcitrant to VIGS studies. However, by using BMV as a vector, a BMV susceptible sorghum variety, $18^{\circ} \mathrm{C}$ for incubating plants, and antisense strand of the target gene fragment, efficient VIGS can still be achieved in sorghum.

\section{Background}

A current challenge to plant scientists is to increase plant productivity in the changing environment with limited resources such as water and fertile land. Sorghum [Sorghum bicolor (L.) Moench.] can grow in dry climatic conditions where summer temperatures are above $20^{\circ} \mathrm{C}[1,2]$. Sorghum is a $\mathrm{C} 4$ annual crop in the grass family and is characterized by its high photosynthetic efficiency. Grain, sweet, and forage type sorghums are all compatible with current agricultural production systems. Sorghum is the fifth most important cereal crop after rice, wheat, maize, and barley [2], with an annual production of about 65.5 million tons from the planted area of 45 million ha worldwide [3]. Sorghum is grown

* Correspondence: ksmysore@noble.org

${ }^{1}$ Noble Research Institute, Ardmore, Oklahoma 73401, USA

Full list of author information is available at the end of the article for food, feed for livestock and fuel. It is a food source for 500 million people in 30 countries [2]. Nearly $80 \%$ of the sorghum-growing area is in developing countries [2]. However, according to Food and Agriculture Organization of the United Nations, in 2014 the USA was the largest producer of sorghum followed by Mexico, Nigeria, Sudan and India [3]. Sorghum production is affected by nearly 150 species of pests, fungus, viruses and parasitic weeds [2]. Also, sorghum is exposed to a variety of abiotic stresses because it is mainly grown on marginal lands [2]. The study of gene function in sorghum will lead to increased productivity of sorghum by improving plant productivity and resistance to biotic and abiotic stresses.

The whole genome sequence of sorghum (BTx623) is available to identify gene sequences that control desirable traits and facilitate molecular breeding (www.phytozome.net/sorghum) [4, 5]. However, it is critical to

(c) The Author(s). 2018 Open Access This article is distributed under the terms of the Creative Commons Attribution 4.0 International License (http://creativecommons.org/licenses/by/4.0/), which permits unrestricted use, distribution, and 
validate the predicted role of the genes. Only a few mutant collections are available for sorghum: a large-scale ethyl methanesulfonate mutagenized library that has nearly 5,000 lines [6] and 5,466 gamma-ray-induced mutant M2 lines [7]. Most of these lines are uncharacterized and therefore the understanding of gene function in sorghum is far from complete.

Virus-induced gene silencing (VIGS) is a quick and robust method to assess the function of a gene or multiple genes by transient post-transcriptional gene silencing [8]. The VIGS mechanism relies on plant antiviral defenses involved in the degradation of viral RNA $[9,10]$. For VIGS, a fragment of a plant gene is inserted into the viral genome. Small interfering (si) RNAs specific to the viral genome and inserted plant gene sequences are generated by Dicer-like protein (DCL). The siRNA integrates with RNA-induced silencing complex (RISC) and targets the viral RNA and specific plant mRNA for degradation [11].

The two critical factors for the success of VIGS are; 1 ) the ability of the virus to infect the plant, 2) the ability of the plant to defend itself from the virus with limited effect on its growth and development. Several VIGS vector systems are available to silence genes in dicotyledons and monocotyledons [8, 12-14]. For monocotyledons, different VIGS vector systems have been developed based on Brome mosaic virus (BMV; [15]), Barley stripe mosaic virus (BSMV;[16, 17]), Bamboo mosaic virus [18], Cymbidium mosaic virus [19], Cucumber mosaic virus [20], Foxtail mosaic virus [21, 22], and Rice tungro bacilliform virus [23].

We tested two VIGS vector systems, BMV and BSMV, to silence genes in sorghum. Ding et al. [15] developed BMV based VIGS vector to silence genes in monocots. Several studies have used BMV based VIGS to silence genes in barley [15], maize [15], rice [15], Festuca arundinacea (Tall Fescue) [24], and sorghum [25, 26]. However, the reported BMV based method for silencing a gene using VIGS in sorghum is cumbersome and is not very efficient with very low frequency of silencing. BSMV was used to silence genes in barley $[16,27]$, wheat $[27,28]$ and in Nicotiana benthamiana [29] but not reported to silence genes in sorghum. Here, we report an improved method to efficiently silence a gene in sorghum using VIGS. We compared several parameters and identified the best environment condition, marker gene, VIGS vector, and sorghum variety for efficient VIGS in sorghum.

\section{Results}

An improved, simple and efficient method to deliver virus into sorghum

BMV-based VIGS vector [15] has been successfully used to silence genes for functional characterization in monocots such as maize [15, 30, 31], barley [15], and rice [15]. The previous method $[25,26]$ used for VIGS in sorghum was not very efficient in our laboratory and needs in vitro RNA synthesis, which is cumbersome and expensive. Here, an easier and an inexpensive method using Agrobacterium-based BMV-VIGS vectors [32, 33] was used to silence genes in sorghum. The BMV genome is made of three positive strand RNAs (RNA 1 [3.2 kb], RNA 2 [2.9 kb] and RNA 3 [2.1 kb]). For VIGS, RNA 1 and RNA 2 are cloned into one Agrobacterium binary vector and RNA 3 into another [32]. A fragment of a plant gene was inserted into RNA 3 as described earlier [15]. We adopted a method that was used earlier to do BMV based VIGS in maize [33]. Briefly, the two Agrobacterium strains containing RNA 1, RNA 2, and RNA 3 were grown to 1.5 O.D $D_{600}$ and mixed in equal amounts to infiltrate into leaves of three weeks old $N$. benthamiana plant for viral multiplication (Fig. 1). The infected $N$. benthamiana leaves were harvested four days after infection and either the sap was extracted or stored at $-80{ }^{\circ} \mathrm{C}$. The sap of the infected $N$. benthamiana was used to inoculate Chenopodium, barley and sorghum plants.

\section{Tolerance of sorghum to BMV decreases at lower temperature}

An important factor for successful VIGS is the ability of the virus to infect and spread within the plant. Contrary to barley and maize, in our greenhouse conditions, we were not able to see visible viral symptoms at high frequency in sorghum after BMV infection at $22^{\circ} \mathrm{C}$ (Fig. 2a). BMV-GFP inoculated maize and barley developed extensive white stripes on the systemic leaves at $22{ }^{\circ} \mathrm{C}$ (Fig. 2a). GFP sequence does not have homology to plant DNA and therefore will not cause any gene silencing in plants. White or yellow stripes are a common symptom due to BMV infection. However, only $~ 10 \%$ of the inoculated sorghum (BTx623 line) plants develop limited white stripes on systemic leaves at $22{ }^{\circ} \mathrm{C}$. Further, inoculation with BMV VIGS construct with a fragment from GFP or $\mathrm{SbChlH}$, or SbPDS genes were not able to produce any viral symptoms or gene silencing in sorghum at $22{ }^{\circ} \mathrm{C}$. In this study, GFP gene fragment was used as a negative control and there are no plant sequences that are homologous to GFP. These constructs produced viral symptoms when infected on Chenopodium and barley at $22{ }^{\circ} \mathrm{C}$ suggesting that the constructs were infectious (Fig. 2b). Interestingly, all the BMV-inoculated BTx623 sorghum plants (100\%) developed disease symptoms when kept at $18{ }^{\circ} \mathrm{C}$ for three days prior to BMV inoculation and three weeks post-BMV inoculation (Fig. 2b). The extent of infection symptoms varied among sorghum plants. These results suggest that sorghum plants incubated at low temperature $\left(18{ }^{\circ} \mathrm{C}\right)$ show better symptoms after BMV challenge than those at $22{ }^{\circ} \mathrm{C}$. Despite viral symptoms at $18{ }^{\circ} \mathrm{C}$, the 

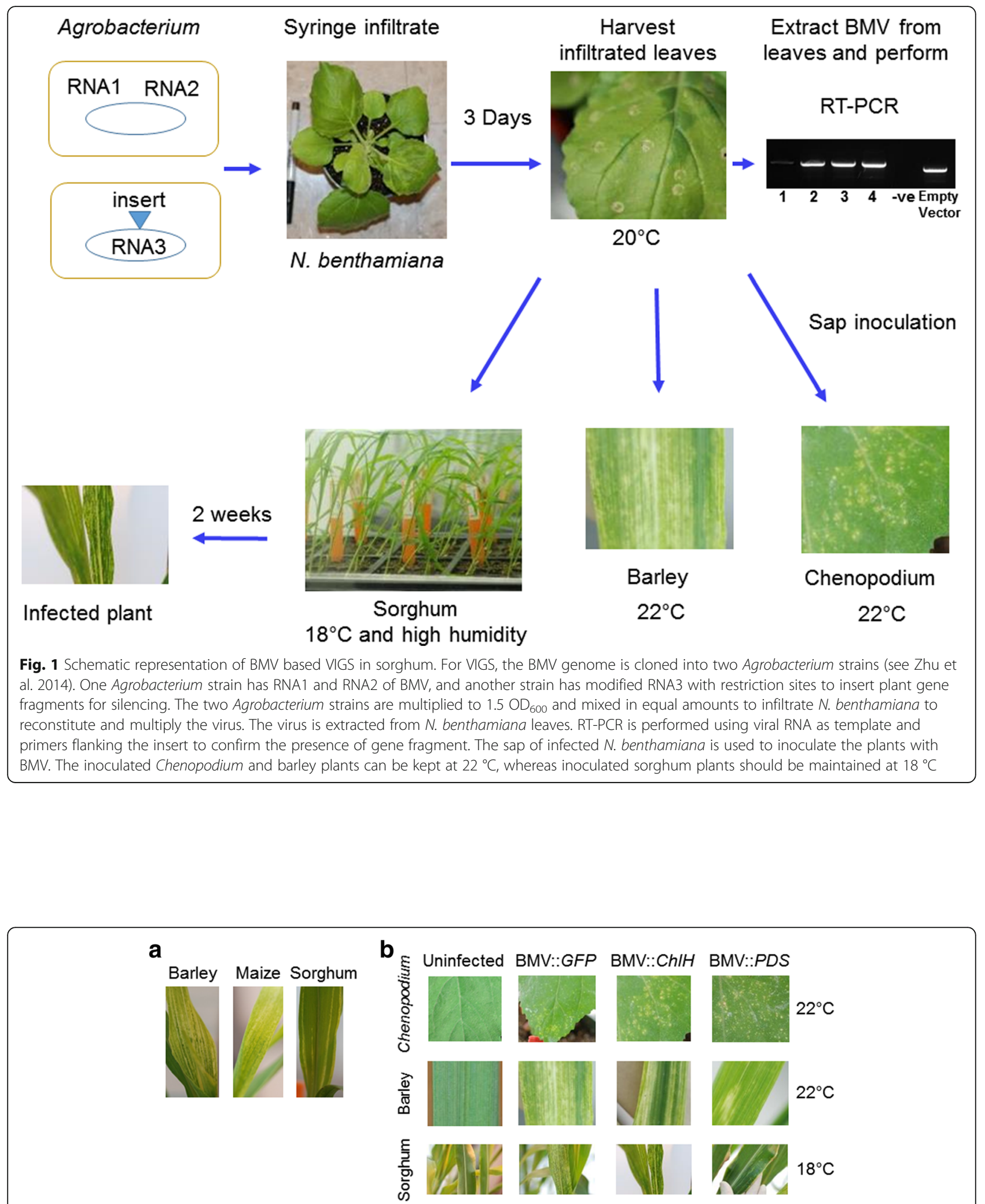

Fig. 2 Sorghum plants incubated at $18{ }^{\circ} \mathrm{C}$ enhance BMV infection. a BMV::GFP infection showed more viral symptoms (bleaching/yellowing) in maize and barley compared to sorghum at $22^{\circ} \mathrm{C}$. b Virus with GFP and sorghum gene fragments: ChlH, or PDS, were grown in N. benthamiana, and sap was isolated and rubbed on Chenopodium, barley, and sorghum plants. BMV was able to infect Chenopodium and barley at $22^{\circ} \mathrm{C}$ and sorghum at $18^{\circ} \mathrm{C}$. 
sorghum plants did not show any visual ChlH or PDS silencing symptoms.

\section{Ubiquitin is a better marker for VIGS in sorghum}

The Phytoene desaturase (PDS) and Magnesium Chelatase subunit $H(\mathrm{ChlH})$ silencing are popularly used as visible markers for VIGS-mediated gene silencing in many plant species [29, 34]. An anti-sense strand of the sorghum $\mathrm{ChlH}$ or PDS gene fragment was used in this study to test the efficiency of BMV-mediated VIGS in sorghum (Fig. 3). Upon inoculation of these constructs to sorghum, at $18{ }^{\circ} \mathrm{C}$, we clearly observed viral symptoms (white stripes) suggesting successful infection in sorghum. Surprisingly, we did not notice whitening of leaves due to $P D S$ silencing in the BMV::anti-PDS inoculated plants or yellowing of leaves due to $\mathrm{ChlH}$ silencing in the BMV::anti-ChlH inoculated plants (Fig. 3a). However, the real-time quantitative RT-PCR (qRT-PCR) data distinctly showed a reduction of transcript level for $\mathrm{ChlH}$ or PDS genes in BMV::anti-ChlH or BMV::anti-PDS inoculated plants, respectively (Fig. 3b). A weak silencing symptom may be masked because the white stripes observed due to the BMV::GFP viral infection are quite similar to PDS or ChlH silencing symptoms. Therefore, we concluded that $P D S$ or $C h l H$ gene silencing are not good visual markers to measure the efficiency of gene silencing in sorghum.

To identify a better visual marker for VIGS in sorghum plants, we developed a BMV-VIGS construct with a fragment of Ubiquitin (Ubiq) gene. We chose to silence Ubiq because it is a crucial protein for plant development and silencing this gene was expected to result in a visual pehnotype. Partial reduction of Ubiq transcripts by transient gene silencing in barley has been shown to cause cell death [35]. Except for the GFP (control) all the gene constructs used were able to silence the target genes when examined by qRT-PCR (Fig. 3b). However, the visible phenotype due to silencing was observed only in the Ubiq silenced plants that showed browning (Fig. 3a). Therefore, we concluded that among all the different silencing markers used, Ubiq is the best visual marker for VIGS in sorghum.

\section{Antisense strand of Ubiq is more efficient in VIGS than the sense strand}

Inserting the antisense strand of a gene in the BMV VIGS vector resulted in a greater degree of silencing compared to using a sense strand in barley [36, 37]. In other studies, the sense or antisense strand of a gene resulted in a similar level of BSMV-based VIGS in barley
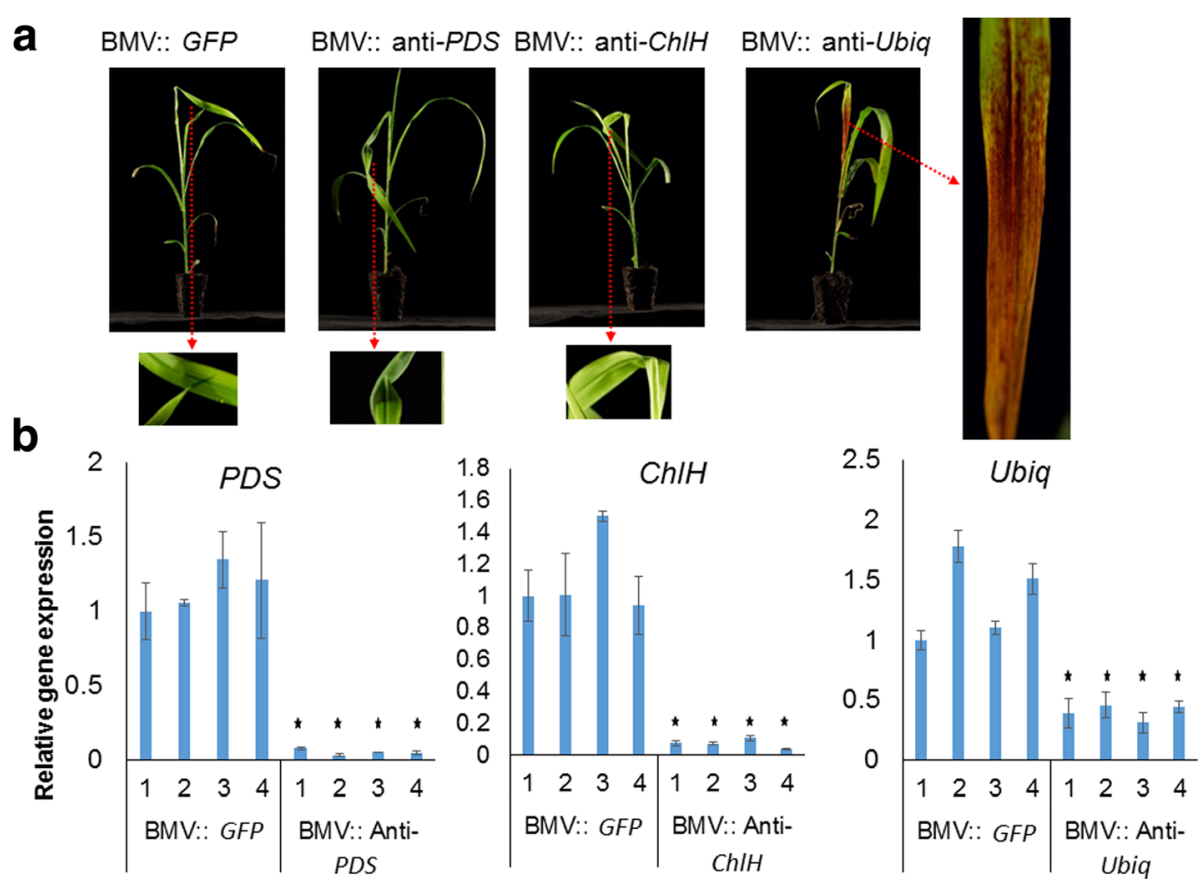

Fig. 3 Ubiquitin silencing is a better marker for VIGS in sorghum. Sorghum plants were rub inoculated with BMV VIGS constructs to silence ChIH, PDS, or Ubiq genes. a Only BMV::anti-Ubiq inoculated plants had a visible phenotype that is different than the control (BMV::GFP). Each viral vector was rub inoculated on 10 sorghum seedlings. Similar results were observed in all the seedlings. A representative image is shown here. $\mathbf{b}$ The RNA was extracted from the second leaf above inoculated leaf of sorghum for gene expression analysis using qRT-PCR. The expression of PDS, ChIH, and Ubiq was significantly lower in the BMV::anti-PDS, BMV::anti-ChIH, and BMV::anti-Ubiq, respectively, compared to control plants (BMV::GFP). Error bars indicate standard deviation of three replicates. The sample was analyzed by student's $t$ test. Asterisks indicate a statistically significant difference compared with the control at $P \leq 0.01$ 
and wheat $[17,38]$. This prompted us to compare the VIGS efficiency of sense versus antisense strand. We generated VIGS construct using sense or antisense of the sorghum Ubiq gene. The same 206 bp fragment of sorghum Ubiq gene was selected to develop both sense and anti-sense constructs. The sequence selected had less homology to other Ubiq gene family members of sorghum.

Sorghum plants infected with either BMV::Ubiq (sense strand) or BMV::anti-Ubiq (antisense strand) developed characteristic brown coloration due to Ubiquitin silencing (Fig. 4a). However, the brown coloration was more widespread in the leaves of BMV::anti-Ubiq inoculated compared to BMV::Ubiq inoculated sorghum (Fig. 4a). Browning was observed in 100\% of BMV::anti-Ubiq infected plants whereas only $73 \%$ in BMV::Ubiq infected plants. We further quantified the level of BMV in the infected $N$. benthamiana that was used to inoculate sorghum plants (Fig. 4b). Antibody specific to the coat protein of BMV was used in the western blot to quantify the virus. When normalized to Actin protein, the levels of BMV was relatively more in the BMV::Ubiq infected compared to the BMV::anti-Ubiq infected N. benthamiana plants (Fig. 4b and Additional file 1: Figure S1). Similar amounts of $N$. benthamiana leaf sap were used to inoculate sorghum. However, BMV amount in the BMV::anti-Ubiq inoculated sorghum plants was slightly ( $\sim 25 \%$ after normalization to control) more compared to
BMV::Ubiq inoculated plants (Fig. 4b and Additional file 1: Figure S1).

Further, the presence of the Ubiq gene fragment (insert) in the BMV VIGS vector isolated from $N$. benthamiana and sorghum inoculated plants was tested. RT-PCR was performed using primers specific to BMV RNA 3 flanking the insertion site. In $N$. benthamiana, the virus with insert was more compared to the virus without the insert in both BMV::Ubiq and BMV::anti-Ubiq infected plants. In sorghum, the virus with insert was more in the BMV::anti- $U$ biq inoculated plants when compared to BMV::Ubiq inoculated plants. Interestingly, the virus without the insert was more in the sorghum plants inoculated with BMV::Ubiq. These results indicate that in sorghum, antisense strand of Ubiq fragment is more stable than sense strand in BMV VIGS vector. However, more experiments with other gene fragments will be needed to conclude that antisense strand will be more efficient for VIGS in sorghum.

\section{BTx623 is a good sorghum variety for BMV-based VIGS}

As mentioned above, one of the important requirements for VIGS is the ability of the virus to infect and multiply in a given plant. Frequency of sorghum infection by BMV, especially at $22{ }^{\circ} \mathrm{C}$ and above, was quite low. We speculated that variations in recalcitrance to BMV infection might exist among different genotypes/varieties of sorghum. Therefore, 67 sorghum genotypes/cultivars a
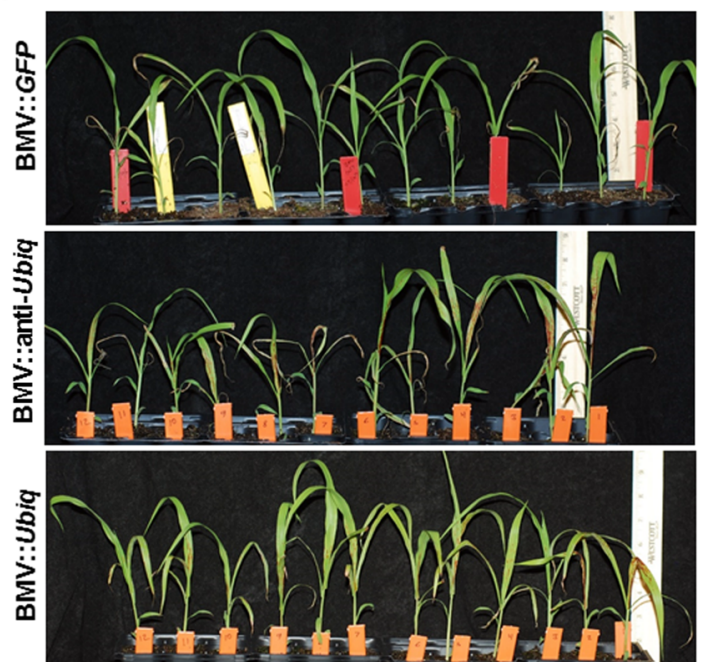

b

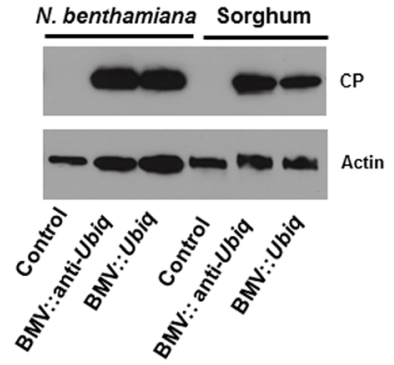

C

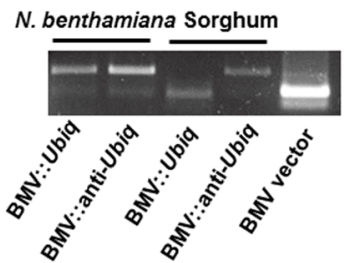

Fig. 4 BMV VIGS is more efficient when an anti-sense strand of the gene fragment is used for silencing. Two-week old sorghum plants were rub inoculated with BMV containing sap of N. benthamiana. a The BMV::Ubiq and BMV::anti-Ubiq infected plants had a varying degree of Ubiq silencing. The extent of silencing was more severe in the BMV:anti-Ubiq infected plants. The experiment was repeated three times with similar results. $\mathbf{b}$ BMV level was analyzed by western blot using an antibody against BMV coat protein. BMV level was more in the sorghum plants infected with BMV: antiUbiq compared to BMV::Ubiq. c RT-PCR was performed to detect the presence of gene fragment in the BMV RNA3 using primers flanking the insert. The BMV with insert was present in the sorghum infected with BMV:anti-Ubiq but not detectable in sorghum infected with BMV::Ubiq. For RT-PCR and western blot, leaves of three $\mathrm{N}$. benthamiana plants or 20 sorghum plants per sample were pooled to extract the virus 
were screened to identify a highly susceptible genotype/ variety to BMV. Two-week old sorghum plants were rub inoculated with BMV::GFP. Three days before rub inoculation, the plants were placed in a growth chamber at $18{ }^{\circ} \mathrm{C}$ and $12 / 12 \mathrm{~h} \mathrm{light/dark}$ cycle. After inoculation, the plants were maintained at $18{ }^{\circ} \mathrm{C}$ for four weeks. At four weeks post-inoculation, the systemic leaves (two leaves above the rub-inoculated leaf) were examined for white stripes (BMV infection symptom). The percentage of inoculated plants showing infection symptoms was recorded (Additional file 2: Table S1). Six genotypes/cultivars with varying degrees of susceptibility to BMV were selected for re-examination by inoculating them with BMV::GFP and BMV::anti-Ubiq (Table 1). Based on the screening, BTx623 was identified as the most susceptible variety for both the constructs of BMV used (Table 1).

Non-uniform silencing in sorghum correlates with uneven distribution of BMV in the infected leaf

Uniform silencing of a gene is observed in leaves of $N$. benthamiana by Tobacco rattle virus -based VIGS [34]; in leaves of soybean, $N$. benthamiana, and pea (Pisum sativum) by Apple latent spherical virus (ALSV) -based VIGS [39]; and in leaves of barley by BSMV based VIGS [29]. However, uniform silencing of Ubiq in sorghum by BMV based VIGS vector is rare. To determine the biological reason for this phenomenon, the presence of BMV in the cells of the infected leaf was observed using a probe designed to hybridize the RNA encoding the coat protein of the virus. The in situ hybridization results suggested that the viral RNA was distributed unevenly throughout the leaf with more concentration around the vascular tissue (Fig. 5a). However, the viral RNA was not present in all the cells of infected vascular tissue (Fig. 5b). These results suggest that the non-uniform silencing in sorghum leaves upon BMV infection is due to the uneven spread of the virus in the leaves.

\section{VIGS can be achieved in sorghum floret}

Determination of gene function in the seed and embryo is crucial to study some traits such as yield. VIGS has been used to study gene function in the inflorescence of soybean and wheat $[27,40,41]$. Silencing of a sorghum gene in the inflorescence cannot be achieved by inoculating leaves with BMV-VIGS construct because VIGS is transient. Therefore, a technique was developed to silence genes in the sorghum inflorescence tissue. Sorghum variety Topper 76 was selected as it is susceptible to BMV and also smaller in size that makes it convenient for inoculation and to grow in the greenhouse. Sorghum inflorescence was rub-inoculated with the sap of $N$. benthamiana that was infected with BMV::anti-Ubiq construct. The inoculated plants were kept at $18{ }^{\circ} \mathrm{C}$ in the dark and humid environment for two days before transferring to a greenhouse with $22{ }^{\circ} \mathrm{C}$ temperature. Keeping plants longer in $18{ }^{\circ} \mathrm{C}$ chamber with $70 \%$ humidity at the flowering stage resulted in leaf damage. The browning due to Ubiq gene silencing was clearly observed in the sorghum floret, rachilla, and rachis (Fig. 6). Initially, silencing was observed in a small area but over time the area of inflorescence showing silencing phenotype increased. These preliminary results suggest that BMV-mediated VIGS can be used to silence genes in reproductive tissue of sorghum.

\section{BSMV-mediated VIGS was not observed in sorghum line BTx623}

In addition to BMV, Barley stripe mosaic virus (BSMV) was also tested as a potential VIGS vector for sorghum. BSMV is popularly used to silence genes in barley and wheat [29]. Agrobacterium-based [29] and in vitro viral RNA synthesis based [38] BSMV-VIGS systems are available. We used Agrobacterium-based BSMV-VIGS system to silence ChlH, PDS, and GFP (control) in sorghum (BTx623). The BSMV genome ( $\alpha-$ RNA, $\beta$-RNA, and $\gamma-$ RNA) is cloned into three different Agrobacterium vectors.

Table 1 A representative list of sorghum genotypes/varieties showing varying degrees of susceptibility to BMV

\begin{tabular}{lll}
\hline $\begin{array}{l}\text { Sorghum } \\
\text { bicolor } \\
\text { genotype }^{\text {a }}\end{array}$ & Percent of plants showing disease phenotype after BMV challenge \\
\cline { 2 - 3 } BTx623 $^{\text {BMV::GFP }}$ & BMV::anti-Ubiq $^{\text {B }}$ & 100.00 \\
Rio & 100.00 & 83.33 \\
Topper 76 & 100.00 & 61.54 \\
Pl 641817 & 80.00 & 50.00 \\
N122B & 54.77 & 33.33 \\
N588 & 41.67 & 16.67 \\
\hline
\end{tabular}

${ }^{\mathrm{a}}$ More than ten plants of each line were used for screening.

${ }^{\mathrm{b}}$ Two weeks old sorghum plants were rub inoculated with BMV::GFP or BMV::anti-Ubiq and plants showing BMV infection symptom were scored three weeks after inoculation. 

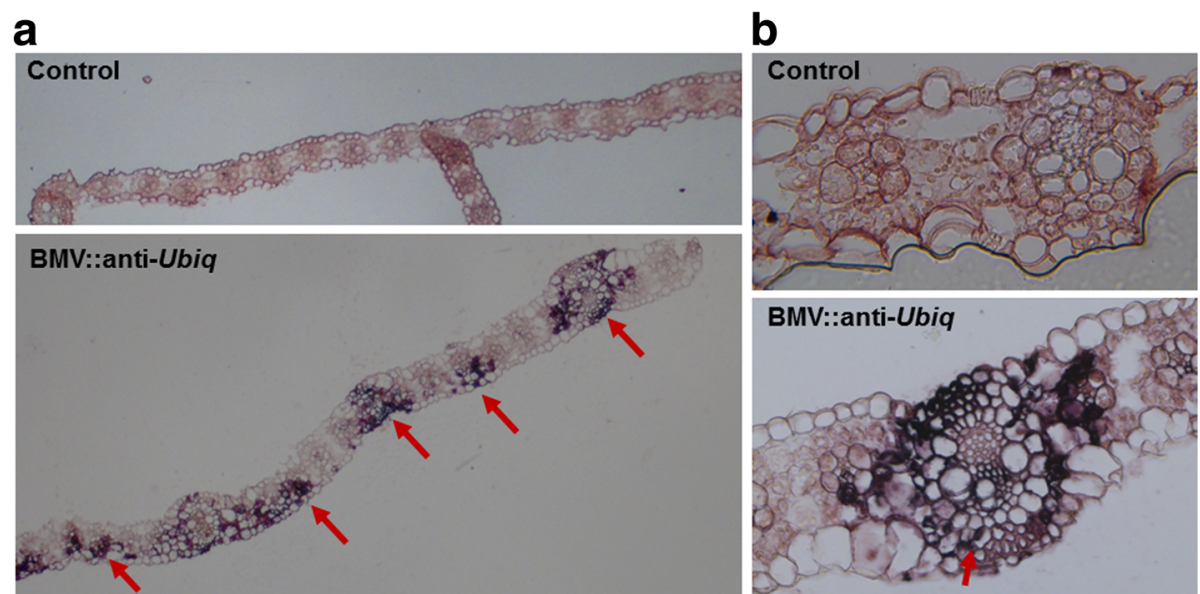

Fig. 5 In situ hybridization shows the uneven spread of BMV virus in sorghum. In situ hybridization probe was designed to hybridize the RNA encoding BMV coat protein. The purple pigment (red arrows) indicates the presence of the virus near veins of the infected leaf. The absence of purple color in the uninfected control sorghum plant indicate a lack of virus. The image was taken with a Nikon TE300 microscope. a Sorghum leaf segment observed by the 10X magnification of the Nikon TE300 microscope. $\mathbf{b}$ The sorghum leaf segment observed by the 20X magnification of the microscope. A similar pattern was seen in two biological replicates

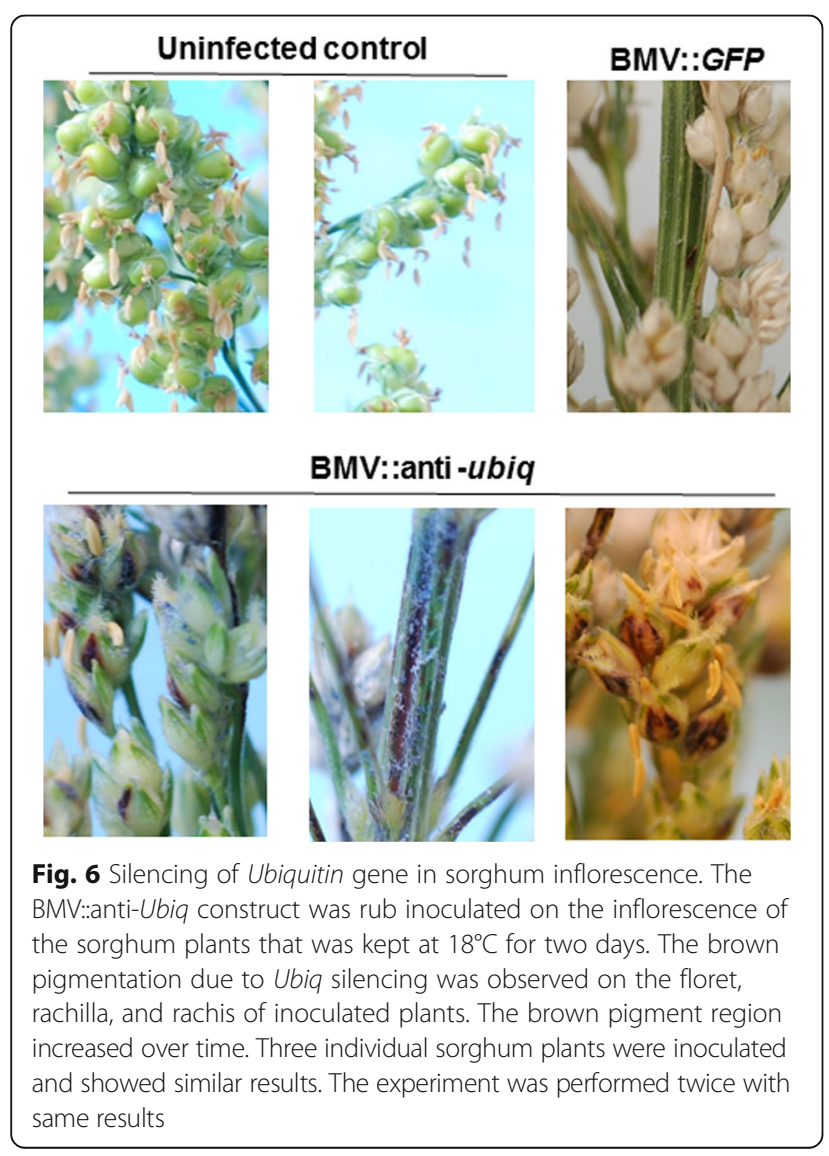

A fragment of plant gene was inserted into $\gamma-$ RNA as described earlier [29]. All the three Agrobacterium strains containing $\alpha-$ RNA, $\beta$-RNA, and $\gamma$-RNA were grown to 1.5 O. $\mathrm{D}_{600}$, mixed in equal amounts and infiltrated into three weeks old $N$. benthamiana leaves for viral multiplication. The infected $N$. benthamiana leaves were harvested after four days and were used to extract sap for infecting Chenopodium, barley and sorghum plants. All the BSMV-VIGS constructs generated were able to infect Chenopodium and barley successfully (Fig. 7a, and 7b). The leaves of barley infected with BSMV::ChlH turned yellow, a phenotype commonly observed upon silencing of $\mathrm{ChlH}$ gene (Fig. 7b; [29]). However, we could not observe any viral symptoms in BTx623 cultivar of sorghum plants inoculated with BSMV. Non-inoculated and inoculated sorghum plants kept at $18{ }^{\circ} \mathrm{C}$ looked similar even 40 days after BSMV inoculation (Fig. 7c). Therefore, we concluded that BSMV based VIGS is not efficient in BTx623 cultivar of sorghum.

\section{Discussion}

The annotated sorghum BTx623 genome has 34,211 protein-encoding loci as reported in Phytozome 11 (https://phytozome.jgi.doe.gov). A robust method is needed to study gene function to improve agronomic traits. Unlike Arabidopsis and rice, stable T-DNA insertion mutants are not available in sorghum. Genetic manipulation of sorghum to generate knockdown/knockout or overexpressor lines is cumbersome requiring several months and a tissue culture facility [42]. VIGS is an alternative method for the functional study of a gene in sorghum. In the present study, we report an improved method of VIGS in sorghum. 
a

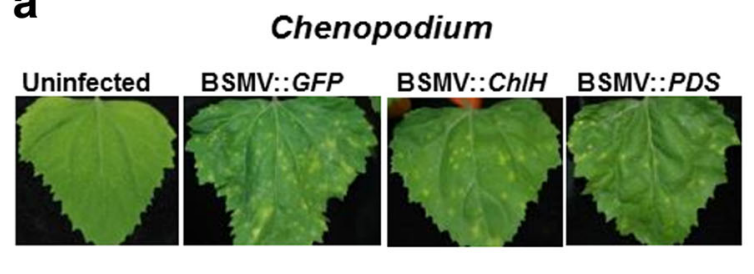

b

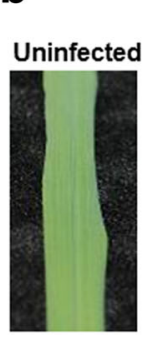

Barley

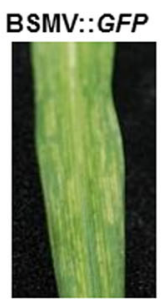

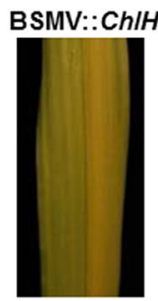

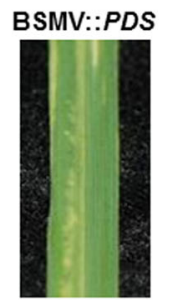

c

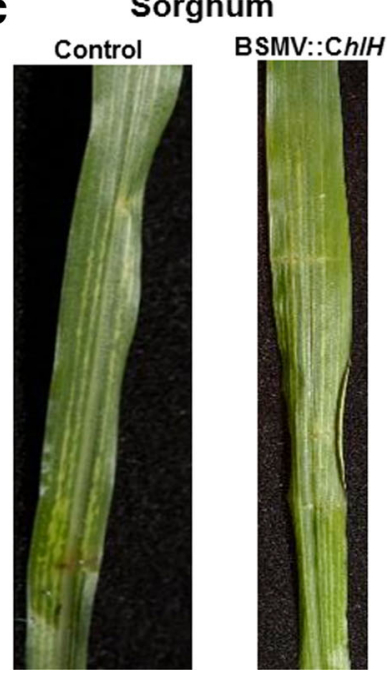

Fig. 7 Sorghum plants are resistant to BSMV infection. Sap from the BSMV inoculated N. benthamiana plants was used to rub inoculate Chenopodium, barley, and sorghum. a The Chenopodium plants showed lesions suggesting that the virus is infectious. $\mathbf{b}$ The white stripes in the systemic leaves of inoculated barley plants suggest successful infection by the virus at $22^{\circ} \mathrm{C}$. The yellowing of the BSMV:: ChIH infected plants indicate successful silencing of the ChlH gene in barley. c Sorghum plants kept at either $20^{\circ} \mathrm{C}$ or $18{ }^{\circ} \mathrm{C}$ had no visible symptoms even 40 days after inoculation with BSMV. White stripes observed on leaves of both uninoculated control and BSMV inoculated sorghum plants is not due to virus infection. The experiment was performed with three barley, two Chenopodium, and 20 sorghum plants. The experiment was repeated twice. Similar results were observed in all the replicates of both the experiments

The first step for successful VIGS is to infect a plant with the virus. At ambient greenhouse growth condition $\left(22{ }^{\circ} \mathrm{C}\right)$ the infectivity of BMV on sorghum occurs at low frequency (Fig. 2a and 2b). Many studies have reported increased infectivity of virus on plants and animals at a lower temperature than the ambient temperature [43, 44]. Increased viral infectivity at a lower temperature is suggested to be due to reduced antiviral defense of the host [43-45]. In addition, lower temperatures have been shown to enhance VIGS phenotype in several dicotyledonous plant species while higher temperature have been shown to increase viral genome silencing [39, 44, 46-48]. In addition, lower light intensity $(<300 \mu \mathrm{E} / \mathrm{m} 2 / \mathrm{s})$ and temperature $\left(25^{\circ} \mathrm{C}\right)$ have more efficient systemic silencing compared to higher light intensity $(\geq 450 \mu \mathrm{E} / \mathrm{m} 2 / \mathrm{s})$ and temperature $\left(30{ }^{\circ} \mathrm{C}\right)$ [49]. Our study showed that higher frequency of infection by BMV could be achieved by infecting sorghum plants kept at $18{ }^{\circ} \mathrm{C}$. Similarly, in wheat, BSMV based VIGS showed better silencing phenotype at lower temperatures of $18-22{ }^{\circ} \mathrm{C}$ [50]. However, several reports in monocotyledonous plants have shown that higher temperature increases viral infection. In barley, BSMV-mediated VIGS provided better results at $20-24{ }^{\circ} \mathrm{C}$ when compared to 16 or $28{ }^{\circ} \mathrm{C}$ [38]. In addition, Ding et al. [51] showed that in barley at lower temperatures (24/ $20{ }^{\circ} \mathrm{C}$, day/night), the BMV virus infected and accumulated mainly in and near vascular cells and limited invasion of vascular tissue was observed. These results suggest that the effect of temperature on VIGS varies among plant-virus combinations and therefore it is important to optimize temperature conditions for every VIGS vector-plant combination studied.

The photobleaching and yellowing of leaves due to $P D S$ and $\mathrm{ChlH}$ silencing, respectively, is used as an indicator of VIGS in dicotyledon and monocotyledon plants [34]. Surprisingly, $P D S$ or $\mathrm{ChlH}$ gene silencing in sorghum by BMV-mediated VIGS did not result in distinctly visible phenotype in sorghum. However, white/yellow stripes on the leaves due to virus infection were visible (Fig. 3a). PDS or $\mathrm{ChlH}$ silencing is therefore not a suitable VIGS marker in sorghum. We propose several reasons why PDS and $\mathrm{ChlH}$ are not suitable VIGS markers in sorghum. 1) The white phenotype due to $P D S$ silencing is similar to virus infection due to BMV infection, 2) the $\mathrm{PDS} / \mathrm{ChlH}$ silencing phenotype is also similar to nitrogen deficiency symptom in sorghum, 3) sorghum is probably tolerant to reduced levels of PDS and $\mathrm{ChlH}$ proteins and therefore doesn't show any visible symptoms.

The housekeeping gene Ubiq is essential for cellular function by targeting proteins for degradation. Therefore, Ubiq gene silencing is expected to cause significant cellular dysfunction leading to cell death, which will be distinctly visible. Partially reducing Ubiq expression level by transient gene silencing in barley lead to cell death [35]. Consistent with these results, our study showed that Ubiq silencing in sorghum by BMV-mediated VIGS showed a clear browning (cell death) phenotype (Fig. 3a). 
Higher efficiency of gene silencing using antisense strand rather than the sense strand of Ubiq during VIGS was observed in our study. BMV is a positive (+) strand RNA virus of family Bromoviridae. In infected host cells, the + strand RNA viruses produce 10 to 100 fold more + strand genomic RNA than the negative (-) strand RNA [52]. Consistently, BMV produces more + strand compared to - strand $[53,54]$. Higher + strand of virus lead to a greater level of viral siRNA from the + strand as observed in plants or animals infected with + RNA viruses $[52,55-58]$. The higher silencing efficiency of the antisense strand of the gene might be due to generation of more siRNA. Also, in sorghum, we detected more retention of the antisense strand than the sense strand in the recombinant BMV. Further, more virus was present in sorghum leaves when the gene fragment was in antisense strand than in sense strand. The cumulative effect of these factors might result in higher VIGS efficiency when antisense strand is used.

VIGS is used to study the function of a gene in the inflorescence of plants. For example, a soybean (Glycine max) seed coat color was modified by silencing a gene using Cucumber mosaic virus based VIGS [40]. Similarly, a $P D S$ gene was silenced in immature pod and seed coat of soybean using ALSV based VIGS by inoculating the first leaf or cotyledon [41]. BSMV based VIGS was used to silence a gene in wheat flowers by inoculating the inflorescence since inoculating seedling was not sufficient to silence a gene in flowers [27]. When the leaves of sorghum seedlings were inoculated with BMV based VIGS, silencing was observed only in up to three new leaves that emerged after inoculation. However, upon infection of the inflorescence with BMV::anti-Ubiq it resulted in the browning of floret, rachilla, and rachis of sorghum (Fig. 6). This is the first report of gene silencing in sorghum inflorescence using VIGS.

\section{Conclusion}

Several VIGS vectors are available to silence genes in monocots. At least two studies report the use of BMV-based VIGS to silence genes in sorghum [25, 26]. The silencing efficiency up to $50 \%$ was achieved in few plants (low frequency) using this method $[25,26]$. Here, we tested several parameters to develop a more efficient BMV based VIGS for sorghum. The virus was multiplied in $N$. benthamiana as done in maize VIGS studies with this vector [33] and unlike in Martin et al., [26] and Biruma et al., [25], where BMV viral mRNA was synthesized in vitro to directly infect sorghum plants. We found three factors that significantly increased VIGS efficiency and frequency in sorghum. 1) Using antisense strand of a gene to develop the VIGS construct instead of sense construct. 2) Keeping the sorghum plants at a lower temperature $\left(18{ }^{\circ} \mathrm{C}\right)$ before and after virus inoculation. 3) Using sorghum variety (eg., BTx623) that allows more BMV multiplication. Further, we identified an appropriate marker gene (Ubiq) that shows clear visible symptoms when silenced. In addition to an improved method for VIGS in sorghum, we also showed that we could silence genes in the reproductive tissue of sorghum by using BMV-based VIGS. An efficient BMV-based VIGS will give a significant boost to gene functional analysis related studies in sorghum.

\section{Methods}

Plant growth, inoculation and disease assessment

Sorghum, barley, Chenopodium, and N. benthamiana plants were grown in a greenhouse at $22{ }^{\circ} \mathrm{C}$ day and night temperature. All plants were grown in the Metromix 360. Leaves of three weeks old $N$. benthamiana plants were infiltarted with disarmed Agrobacterium tumefaciens strain GV2260 containing the recombinant BMV vectors within the T-DNA using a needless syringe. Inoculated (as described below) barley, Chenopodium, and $N$. benthamiana plants were kept in the greenhouse with $16 \mathrm{~h}$ photoperiod and $22{ }^{\circ} \mathrm{C}$ temperature. Sorghum plants were maintained in a growth chamber at $18{ }^{\circ} \mathrm{C}$, $12 \mathrm{~h}$ photoperiod, $70 \%$ humidity, and 150-200 $\mu$ molm $^{-2} \mathrm{~s}^{-1}$ light intensity 3 days before inoculation and 3 weeks post inoculation. The BMV infection symptom was assessed by counting the plants with white stripes in the second leaves above the infected leaves at 4 weeks after rub inoculation.

\section{Developing BMV VIGS constructs}

The RNA from the 3 weeks old sorghum plants was used to generate cDNA to PCR amplify gene fragments for VIGS construct generation. The $5^{\prime}$ end of the primers was designed to have NcoI or AvrII restriction enzyme sites for restriction digestion and cloning into BMV-VIGS vector $[15,33]$. The primer sequences used for the experiment are listed in Additional file 3: Table S2. The size of the insert was from 200 to 400 base pairs. After cloning the insert into the vector, BMV3 F primer (Additional file 3: Table S2) was used for sequencing. The plasmid with the insert was transformed into $A$. tumefaciens strain GV2260.

\section{Plant inoculation with BMV}

Two Agrobacterium strains, one with RNA1 and RNA2 of BMV and another with RNA3 of the BMV genome were grown overnight in LB medium. The strains were centrifuged and resuspended to $1.5 \mathrm{OD}_{600}$ in $10 \mathrm{mM}$ MES (pH 5.8) and $100 \mathrm{nM}$ acetosyringone. An equal amount of two Agrobacterium strains were mixed and kept in a shaker at room temperature for $3 \mathrm{~h}$ in the dark. The Agrobacterium cocktail was syringe (needless) infiltrated into fully open leaves of $N$. benthamiana grown 
in a greenhouse at $22{ }^{\circ} \mathrm{C}$. $N$. benthamiana leaves were harvested 4 days after infiltration and used to prepare sap as described below or were frozen in liquid nitrogen and stored at $-80{ }^{\circ} \mathrm{C}$ for future use.

The inoculation method is schematically represented in Fig. 1. Sorghum plants were grown in a greenhouse at $22{ }^{\circ} \mathrm{C}$ for 12 days (four-leaf stage). The young plants were then transferred to a growth chamber at $18{ }^{\circ} \mathrm{C}$ and $12 \mathrm{~h} / 12 \mathrm{~h} \mathrm{light/dark}$ cycle for three days. The sap for rub-inoculation was prepared by grinding $2 \mathrm{~g}$ of infected $N$. benthamiana leaf in $1 \mathrm{ml} 10 \mathrm{mM}$ potassium phosphate buffer (pH 6.8) and $100 \mathrm{mg}$ of carborundum abrasive. The sap is rubbed on two to three bottom leaves of sorghum. Inoculated plants were covered with a dome to maintain high humidity and kept in the dark for two days at $18{ }^{\circ} \mathrm{C}$. Inoculated plants were then transferred to $18^{\circ} \mathrm{C}$ growth chamber with $12 \mathrm{~h} / 12 \mathrm{~h}$ light/dark cycle. Similar to sorghum, Chenopodium and barley plants were also inoculated with the sap as described above for sorghum, except that the plants were kept at $22{ }^{\circ} \mathrm{C}$. BSMV inoculation was also done in a similar fashion as BMV.

\section{Quantitative RT-PCR}

Total plant RNA was extracted using a Qiagen RNAeasy kit (www.qiagen.com). The RNA was treated with RNAse-free DNAse I. Superscript III was used to synthesize the first strand of the cDNA and oligo (dT) primer. Quantitative RT-PCR was performed using 10-fold diluted cDNA, SYBR green Real-time PCR Master Mix (www.thermofisher.com), and ABI PRISM 7500 (Applied Biosystems, NY, USA). The transcript level of a gene was normalized with SbActin. Student's $t$-test determined the statistically significant difference between the expression level in the silenced and control plants.

\section{Virus extraction and RT-PCR}

For virus extraction, leaves of $N$. benthamiana were harvested 4 days after inoculation and leaves of sorghum were harvested three weeks post inoculation. BMV was extracted by the polyethylene glycol precipitation method [15]. Phenol/chloroform was used to extract RNA from the purified virus [15]. The first strand of the cDNA was synthesized using Superscript III and random primers. PCR using BMV3 forward and reverse primers (Additional file 3: Table S2) that flank the insert DNA in the RNA3 of the virus was performed.

\section{BMV quantification}

Total protein was extracted from the leaves of infected $N$. benthamiana and sorghum using extraction buffer containing $1 \mathrm{mM} \mathrm{MgCl} 2,220 \mathrm{mM}$ Tris/ $\mathrm{HCl} \mathrm{pH}$ 7.4, 50 $\mathrm{mM} \mathrm{KCl}, 250 \mathrm{mM}$ sucrose, and $10 \mathrm{mM} \beta$ - mercaptoethanol. An equal amount ( $2 \mathrm{ug}$ ) of extracted protein was separated using SDS-PAGE in $12.5 \%$ gels and then subjected to western blot using an antibody specific to BMV coat protein [51].

\section{In situ hybridization}

The BMV RNA3-Insitu-L/R primer pairs (Additional file 3: Table S2) were used in PCR for probe preparation to amplify a 362-bp of the RNA3 of BMV. The PCR probe was labeled with Digoxigenin-11-UTP (Sigma-Aldrich Co. LLC, Mo, USA). Long's protocol (http://www.its.caltech.edu/ plantlab/protocols/insitu.pdf) was used for tissue preparation. Robot GenePaint system (Tecan) was used for pre-hybridization, hybridization, and washing [59]. The section was imaged using the Nikon Eclipse TE 300 (Nikon Inc. NY, USA).

\section{Additional files}

Additional file 1: Figure S1. BMV capsid protein quantification. BMV level was analyzed by western blot using an antibody against BMV coat protein. The capsid protein was normalized with Actin protein of the plants. In N. benthamiana, the BMV level was more in the BMV:: Ubia infected plant compared to BMV:: anti-Ubiq infected plant. In sorghum, BMV level is similar in both BMV:: anti-Ubiq and BMV:: Ubiq infected plants. (PDF $63 \mathrm{~kb}$ )

Additional file 2: Table S1. List of sorghum genotypes/varieties showing BMV infection. (XLSX $11 \mathrm{~kb}$ )

Additional file 3: Table S2. List of primers used in this study. (XLSX 12 kb)

\section{Abbreviations}

VIGS: Virus-induced gene silencing; BMV: Brome mosaic virus; BSMV: Barley stripe mosaic virus; Ubiq: Ubiquitin; $\mathrm{ChlH}$ : Magnesium chelatase subunit $\mathrm{H}$; PDS: Phytoene desaturase; SiRNA: Small interfering RNA

\section{Acknowledgments}

We thank Dr. X. S. Ding and Dr. Rick Nelson (Noble Research Institute) for providing the BMV VIGS vectors, an antibody against BMV coat protein, and useful suggestions, and latter providing critical comments on the manuscript. We thank Dr. Andy Jackson from the University of Berkeley for providing us BSMV based VIGS vector and Guifen Li \& Yuhong Tang (Noble Research Institute) for in situ hybridization. We thank Dr. Sally Mackenzie (University of Nebraska-Lincoln) for encouragement and discussion throughout the project. We acknowledge the contribution of Sunhee Oh (Noble Research Institute) for technical assistance. This work was funded by Bill and Melinda Gates

Foundation through the University of Nebraska-Lincoln.

\section{Supporting Information}

The following Supporting Information is available for this article:

\section{Funding}

This work was funded by the Bill and Melinda Gates Foundation through the University of Nebraska-Lincoln.

Availability of data and materials

All the data pertaining to this manuscript provide either in the main manuscript or in the supplemental files that can be accessed from the journal website.

\section{Consent for publication}

Not applicable.

\section{Authors' contributions}

KM and DS designed research; DS and H-KL performed research; ID provided reagents; DS and KM wrote the paper. All authors read and approved the final manuscript. 


\section{Ethics approval and consent to participate}

Not applicable

\section{Competing interests}

The authors declare that they have no competing interests.

\section{Author details}

${ }^{1}$ Noble Research Institute, Ardmore, Oklahoma 73401, USA. ${ }^{2}$ Department of Agronomy and Horticulture, University of Nebraska-Lincoln, Lincoln, Nebraska 68583, USA.

Received: 4 December 2017 Accepted: 6 June 2018 Published online: 18 June 2018

\section{References}

1. Morris GP, Ramu P, Deshpande SP, Hash CT, Shah T, Upadhyaya HD, et al. Population genomic and genome-wide association studies of agroclimatic traits in sorghum. Proc Nat Acad Sci. 2018;110:453-8.

2. SrinivasaRao P, Belum VSR, Nagaraj N, Hari DU. Sorghum production for diversified uses. In: Genetics, Genomics and Breeding of Sorghum. CRC Press. 2014;2014:1-27.

3. FAOSTAT. Production of crops. Food and Agriculture Organization of the United Nations (FAO) Statistics Division. Available from: http://faostat3.fao. org/download/Q/QC/E (Accessed 18.05.2015) 2015.

4. Mace ES, Tai S, Gilding EK, Li Y, Prentis PJ, Bian L. Whole-genome sequencing reveals untapped genetic potential in Africa's indigenous cereal crop sorghum. Nat. Commun. 2013:4:2320

5. Paterson AH, Bowers JE, Bruggmann R, Dubchak I, Grimwood J, Gundlach H. The Sorghum bicolor genome and the diversification of grasses. Nature. 2009:457:551-6.

6. Xin Z, Wang M-L, Chopra S, Wang P. Gene mutagenesis systems and resources for the Saccharinae. In: Paterson $\mathrm{AH}$, editor. Genomics of the Saccharinae. New York: Springer Verlag; 2013. p. 169-85.

7. Ordonio R, Ito Y, Morinaka Y, Sazuka T, Matsuoka M. Molecular breeding of Sorghum bicolor, a novel energy crop. In: Kwang WJ, editor. International Review of Cell and Molecular Biology. Academic Press; 2016. p. 221-57.

8. Senthil-Kumar M, Mysore KS. New dimensions for VIGS in plant functional genomics. Trends Plant Sci. 2011;16:656-65.

9. Ma X, Nicole M-C, Meteignier L-V, Hong N, Wang G, Moffett P. Different roles for RNA silencing and RNA processing components in virus recovery and virus-induced gene silencing in plants. J Exp Bot. 2014;66:919-32.

10. Baulcombe DC. Fast forward genetics based on virus-induced gene silencing. Curr Opin Plant Biol. 1999;2:109-13.

11. Baulcombe DC. RNA silencing in plants. Nature. 2004;432:356-63.

12. Lee WS, Rudd JJ, Kanyuka K. Virus induced gene silencing (VIGS) for functional analysis of wheat genes involved in Zymoseptoria tritici susceptibility and resistance. Fungal Genet Biol. 2015;79:84-8.

13. Lee WS, Kim E, Kosack H. Kanyuka, In plants transient expression systems for monocots. In: Azhakanandam K, Silverstone A, Daniell H, Davey MR, editors. Recent advancements in gene expression and enabling technologies in crop plants. Springer; 2015. p. 391-422.

14. Cheuk A, Houde M. A rapid and efficient method for uniform gene expression using the Barley stripe mosaic virus. Plant Methods. 2017;13:24.

15. Ding XS, Schneider WL, Chaluvadi SR, Mian MA, Nelson RS. Characterization of a Brome mosaic virus strain and its use as a vector for gene silencing in monocotyledonous hosts. Mol Plant-Microbe Interact. 2006;19:1229-39.

16. Holzberg S, Brosio P, Gross C, Pogue GP. Barley stripe mosaic virus-induced gene silencing in a monocot plant. Plant J. 2002;30:315-27.

17. Scofield SR, Huang L, Brandt AS, Gill BS. Development of a virus-induced gene-silencing system for hexaploid wheat and its use in functional analysis of the Lr21-mediated leaf rust resistance pathway. Plant Physiol. 2005;138; 2165-2173.

18. Liou M-R, Huang Y-W, Hu C-C, Lin N-S, Hsu Y-H. A dual gene-silencing vector system for monocot and dicot plants. Plant Biotechnol J. 2014;12 330-43.

19. Lu H-C, Chen H-H, Tsai W-C, Chen W-H, Su H-J. Chang DC-N, Yeh H-H. Strategies for functional validation of genes involved in reproductive stages of orchids. Plant Physiol. 2007;143:558-69.

20. Wang R, Yang X, Wang N, Liu X, Nelson RS, Li W, et al. An efficient virusinduced gene silencing vector for maize functional genomics research. Plant J. 2016;86:102-15.
21. Liu N, Xie K, Jia Q, Zhao J, Chen T, Li H, Wei X, Diqo X, Hong Y, Liu Y. Foxtail Mosaic Virus- induced gene silencing in monocot plants. Plant Physiol. 2016; 171(18):1-1807.

22. Mei Y, Zhang C, Kernodle BM, Hill JH, Whitham SAA. Foxtail mosaic virus vector for virus-induced gene silencing in maize. Plant Physiol. 2016;171: 760-72.

23. Purkayastha A, Mathur S, Verma V, Sharma S, Dasgupta I. Virus-induced gene silencing in rice using a vector derived from a DNA virus. Planta. 2010;232: 1531-40.

24. Scofield SR, Nelson RS. Resources for virus-induced gene silencing in the grasses. Plant Physiol. 2009;149:152-7.

25. Biruma M, Martin T, Fridborg I, Okori P, Dixelius C. Two loci in sorghum with NB-LRR encoding genes confer resistance to Colletotrichum sublineolum. Theor App Genet. 2012;124:1005-15.

26. Martin T, Biruma M, Fridborg I, Okori P. Dixelius C. A highly conserved NBLRR encoding gene cluster effective against Setosphaeria turcica in sorghum. BMC Plant Biol. 2011;11:151.

27. Ma M, Yan Y, Huang L, Chen M, Zhao H. Virus-induced gene-silencing in wheat spikes and grains and its application in functional analysis of HMWGS-encoding genes. BMC Plant Biol. 2012;12:141.

28. Tufan HA, Stefanato FL, McGrann GR, MacCormack R, Boyd LA. The Barley stripe mosaic virus system used for virus-induced gene silencing in cereals differentially affects susceptibility to fungal pathogens in wheat. J Plant Physiol. 2011;168:990-4.

29. Yuan C, Li C, Yan L, Jackson AO, Liu Z, Han C, et al. A high throughput Barley stripe mosaic virus vector for virus induced gene silencing in monocots and dicots. PLoS One. 2011;6:e26468.

30. van der Linde K, Kastner C, Kumlehn J, Kahmann R, Doehlemann G Systemic virus-induced gene silencing allows functional characterization of maize genes during biotrophic interaction with Ustilago maydis. New Phytol. 2011;189:471-83.

31. Cao Y, Shi YAN, Li Y, Cheng Y, Zhou TAO, Fan Z. Possible involvement of maize Rop1 in the defence responses of plants to viral infection. Mol Plant Pathol. 2012;13:732-43.

32. Ding XS, Mannas SW, Bishop BA, Rao X, Lecoultre M, Kwon S, Nelson RS. An improved Brome mosaic virus silencing vector: Greater insert stability and more extensive VIGS. Plant Physiol. 2017; https://doi.org/10. 1104/pp.17.00905.

33. Zhu M, Chen Y, Ding XS, Webb SL, Zhou T, Nelson RS, Fan Z. Maize Elongin $C$ interacts with the viral genome-linked protein, VPg, of Sugarcane mosaic virus and facilitates virus infection. New Phytol. 2014;203:1291-304.

34. Senthil-Kumar M, Mysore KS. Tobacco rattle virus-based virus-induced gene silencing in Nicotiana benthamiana. Nat Protocols. 2014;9:1549-62.

35. Dong W, Nowara D, Schweizer P. Protein polyubiquitination plays a role in basal host resistance of barley. Plant Cell. 2006;18:3321-31.

36. Pacak A, Strozycki PM, Barciszewska-Pacak M, Alejska M, Lacomme C, Jarmolowski A, et al. The brome mosaic virus-based recombination vector triggers a limited gene silencing response depending on the orientation of the inserted sequence. Arch Virol. 2010;155:169-79.

37. Ramanna H, Ding XS, Nelson RS. Rationale for developing new virus vectors to analyze gene function in grasses through virus-induced gene silencing. Methods Mol Biol. 2013;975:15-32.

38. Bruun-Rasmussen M, Madsen $C T$, Jessing S, Albrechtsen M. Stability of Barley stripe mosaic virus-induced gene silencing in barley. Mol Plant-Microbe Interact. 2007:20:1323-31.

39. Igarashi A, Yamagata K, Sugai T, Takahashi Y, Sugawara E, Tamura A, et al. Apple latent spherical virus vectors for reliable and effective virus-induced gene silencing among a broad range of plants including tobacco, tomato, Arabidopsis thaliana, cucurbits, and legumes. Virology. 2009;386:407-16.

40. Nagamatsu A, Masuta C, Senda M, Matsuura H, Kasai A, Hong J-S, et al. Functional analysis of soybean genes involved in flavonoid biosynthesis by virus-induced gene silencing. Plant Biotechnol J. 2007;5:778-90.

41. Yamagishi N, Yoshikawa N. Virus-induced gene silencing in soybean seeds and the emergence stage of soybean plants with Apple latent spherical virus vectors. Plant Mol Biol. 2009;71:15-24

42. Wu E, Lenderts B, Glassman K, Berezowska-Kaniewska M, Christensen H, Asmus T, et al. Optimized Agrobacterium-mediated sorghum transformation protocol and molecular data of transgenic sorghum plants. In Vitro Cell Dev Biol. 2014;50:9-18.

43. Adelman ZN, Anderson MAE, Wiley MR, Murreddu MG, Samuel GH Morazzani EM, Myles KM. Cooler temperatures destabilize RNA interference 
and increase susceptibility of disease vector mosquitoes to viral infection. PLoS Negl Trop Dis. 2013;7:e2239.

44. Szittya G, Silhavy D, Molnár A, Havelda Z, Lovas Á, Lakatos L, et al. Low temperature inhibits RNA silencing-mediated defence by the control of siRNA generation. EMBO J. 2003;22:633-40.

45. Adelman ZN, Anderson MAE, Morazzani EM, Myles KM. A transgenic sensor strain for monitoring the RNAi pathway in the yellow fever mosquito Aedes aegypti. Insect Biochem Mol Biol. 2008;38:705-13.

46. Chellappan P, Vanitharani R, Ogbe F, Fauquet CM. Effect of temperature on gemini virus induced RNA silencing in plants. Plant Physiol. 2005;138:1828-41.

47. Fu DQ, Zhu BZ, Zhu HL, Zhang HX, Xie YH, Jiang WB, et al. Enhancement of virus-induced gene silencing in tomato by low temperature and low humidity. Mol Cells. 2006;21:153-60.

48. Tuttle JR, Idris AM, Brown JK, Haigler CH, Robertson D. Geminivirusmediated gene silencing from Cotton leaf crumple virus is enhanced by low temperature in cotton. Plant Physiol. 2008;148:41-50.

49. Patil BL, Fauquet CM. Light intensity and temperature affect systemic spread of silencing signal in transient agroinfiltration studies. Mol Plant Pathol. 2015;16:484-94.

50. Cakir C, Tor M. Factors influencing Barley stripe mosaic virus-mediated gene silencing in wheat. Physiol Mol Plant Pathol. 2010;74:246-53.

51. Ding XS, Flasinski S, Nelson RS. Infection of Barley by Brome mosaic virus is restricted predominantly to cells in and associated with veins through a temperature-dependent mechanism. Mol Plant-Microbe Interact. 1999;12: 615-23.

52. Ding S-W. RNA-based antiviral immunity. Nat Rev Immunol. 2010;10:632-44.

53. Janda M, Ahlquist P. RNA-dependent replication, transcription, and persistence of brome mosaic virus RNA replicons in S. cerevisiae. Cell. 1993; 72:961-70.

54. Noueiry AO, Ahlquist P. Brome mosaic virus RNA replication: Revealing the role of the host in RNA virus replication. Annu Rev Phytopathol. 2003;41:77-98.

55. Ho T, Pallett D, Rusholme R, Dalmay T. Wang H. A simplified method for cloning of short interfering RNAs from Brassica juncea infected with Turnip mosaic potyvirus and Turnip crinkle carmovirus. J Virol Methods. 2006;136: 217-23.

56. Molnár A, Csorba T, Lakatos L, Várallyay É, Lacomme C, Burgyán J. Plant virus-derived small interfering RNAs originate predominantly from highly structured single-stranded viral RNAs. J Virol. 2005;79:7812-8.

57. Qi X, Bao FS, Xie Z. Small RNA deep sequencing reveals role for Arabidopsis thaliana RNA-dependent RNA polymerases in viral siRNA biogenesis. PLoS One. 2009;: :e4971.

58. Singh K, Kaur R, Qiu W. New virus discovery by deep sequencing of small RNAs. In: Jin, H, Gassmann W. editors. RNA Abundance Analysis: Methods and Protocols. Totowa, NJ: Humana Press; 2012. p. 177-191.

59. Jackson D. In situ hybridization in plants. In: Bowles DJ, McPherson, M. editors. Molecular Plant Pathology: A Practical Approach. Oxford: Oxford Univ Press; 1991. p. 163-174.

\section{Ready to submit your research? Choose BMC and benefit from:}

- fast, convenient online submission

- thorough peer review by experienced researchers in your field

- rapid publication on acceptance

- support for research data, including large and complex data types

- gold Open Access which fosters wider collaboration and increased citations

- maximum visibility for your research: over $100 \mathrm{M}$ website views per year

At BMC, research is always in progress.

Learn more biomedcentral.com/submissions 